\title{
Analysis of deformation characteristics of Asymmetric Gradient Extrusion in preparing ultra- fine grained bulk materials
}

Junkai Fan ( $\nabla$ junkaifan@hpu.edu.cn )

Henan Polytechnic University

Chengpeng Wang

The Institute of Seawater Desalination \& Multipurpose Utilization

Wu Zhao

Henan Polytechnic University

Wei Liu

Henan Polytechnic University

\section{Research Article}

Keywords: Asymmetric Gradient Extrusion (AGE), deformation characteristics, slip line field, finite element analysis, ultra-fine grained bulk materials

Posted Date: May 14th, 2021

DOI: https://doi.org/10.21203/rs.3.rs-505517/v1

License: (c) (i) This work is licensed under a Creative Commons Attribution 4.0 International License. Read Full License 


\section{Abstract}

A novel method to prepare ultra-fine grained bulk materials, which is named Asymmetric Gradient Extrusion (AGE), is proposed in this article. In AGE, the cross section of extrusion channel is rectangle and two inclined planes are stagger arranged along extrusion direction. To realize repeating extrusion, the thickness of workpiece is restricted to equal the width of channel's outlet. The deformation characteristics of AGE was first theoretically analyzed by slip line field. Then, these results were verified and supplemented by finite element analysis. The results reveal that deformation characteristics of workpiece in channel is largely related to the two inclined planes. Two independent deformation zones (TIDZ) can be formed with increasing distance between the two inclined planes. In addition, the accumulated strain generated in the TIDZ is complementary in value. Furthermore, the shearing effects of workpiece subjected in one pass extrusion has been improved. A more uniform strain distribution and higher shearing effect can be generated in AGE, which make it as a very promising method to produce ultra-fine grained bulk materials.

\section{Introduction}

Ultra-fine grained (UFG) materials, which exhibit excellent mechanical properties, have attracted great interesting during the past decades ${ }^{[1]}$. Important contributions in this context have been made by the development of so-called "top-down" techniques. Severe plastic deformation (SPD) method as one of these techniques is of more interest as it provides UFG or even bulk nanostructured materials (BNMs) with no contamination or porosity ${ }^{[2]}$.

Distinguished with other methods, most of SPD techniques realize grain fragment by repetitive processes. In these processes, the cross-section dimensions of workpiece should be remaining unchanged after each processing cycles. In addition, an aimed very high strain can be accumulated after several cycles which makes the initial grains fragment into ultrafine or nanometric size. Since SPD was firstly introduced as an effective approach to produce UFG metals in $1980 \mathrm{~s}^{[3]}$, enormous efforts have been made to develop SPD techniques, e.g., equal-channel angle pressing (ECAP) ${ }^{[4,5]}$, high pressure torsion (HPT) ${ }^{[6,7]}$, accumulated roll-bonding (ARB) ${ }^{[8,9]}$, multi-directional forging (MDF) ${ }^{[10,11]}$, cyclic extrusioncompression(CEC) ${ }^{[12,13]}$, repetitive corrugation and straightening(RCS) ${ }^{[14,15]}$, twist extrusion (TE) ${ }^{[16,17]}$, constrained groove pressing (CGP) ${ }^{[18,19]}$ and so on.

Direct extrusion is a traditional metal forming method, which can generate a large strain, so that it has the potential to prepare ultrafine grained materials. The possibility of using extrusion to prepare ultrafine grained materials has been verified ${ }^{[20,21]}$. Using high ratio extrusion (HRE) ${ }^{[22]}$ or hydrostatic extrusion with an extra high ratio ${ }^{[23]}$, favorable results in grain refinement were reported. However, these extrusion techniques all change the shape of the material, making it difficult to repeatedly extrude material with the same die. Moreover, the uniformity of strain distribution inside the material after extrusion is poor. 
Therefore, some modifications should be made to overcome these obstacles in extrusion to realize grains fragment.

Based on the conventical direct extrusion, this article proposes a new SPD method, which named Asymmetric Gradient Extrusion (AGE), to produce ultra-fine grained bulk materials. Comparing with traditional extrusion processes, a much more uniform strain distribution can be realized with this new SPD technique. The deformation characters of billet in one pass of AGE is theoretically analyzed by slip line field. In addition, finite element analysis was proceeded to verify the theoretical results and the advantage of this SPD technique in producing ultra-fine grained materials is also emphasized and discussed.

\section{Principle Of The Asymmetric Gradient Extrusion Technique}

In most cases of extrusion, the die apertures are of symmetry character in geometry. Accordingly, deformation zones are formed symmetrically in workpiece and the aimed reduction in cross-section of workpiece can be realized. However, the variation in cross-section brings a huge difficulty in re-extruding workpiece with the same die. To solve this issue, some amendments have been introduced in AGE. Firstly, the cross-section of die aperture and initial billet are all designed with rectangle profile which are shown in Fig. 1(a). Therefore, the deformation of workpiece in channel can be treated approximatively as a plane strain case. The illustration of AGE process on a plane is displayed in Fig. 1(b). To make sure the shape of workpiece unchanged after extrusion, the thickness of initial billet is constrainedly equal to the width of channel outlet. As shown in Fig. 1(b), the extruded workpiece has the same shape and dimensions with its initial counterpart. Therefore, the repetitive extrusion of workpiece can be realized, and an aimed strain can be gained in workpiece with several cycles.

Another modification has been introduced in AGE is the asymmetric distribution of two extrusion bevels in channel which are generally symmetric designed in conventional extrusion. A vertical distance $h$ is arranged between the two extrusion bevels, which has great effect on the deformation behaviors of workpiece in extrusion. Depends on the extrusion bevels, two deformation zones (DZs) would be formed, and the parameter $h$ defines the independence or interaction of this two DZs. The influence of parameter $h$ would be studied and discussed in the next parts. Besides $h$, the angles $a$ and $\beta$ of two extrusion bevels also can be assigned independently. These two parameters are largely related to the characters of deformation fields around the two extrusion bevels. However, the same angles are used in this article. The influence of the two angles and parameters optimization of AGE will be dealt with in our next work.

\section{Mechanism Of Grain Fragmentation}

According to the characteristics of metal flow in channel, the workpiece can be divided into five parts, which are schematic illustrated in Figure 2. In region $\nabla$, the material, assumed to be rigid, moves downward with a constant velocity $V_{\square}$, which is equal to the punch velocity. The material undergoes plastic deformation in region $\nabla$ and region $\nabla$. Depends on the value of parameter $h$ assigned, the two 
deformation regions $₫$ and $\otimes$ can be interacted in region $\otimes$ or totally independent. However, to realize our goal, the parameter $h$ should be large enough to separate the two plastic deformation regions. Therefore, the material in region $\mathbb{Q}$ is flow rigidly. In region $\square$, the material moves to the exit of the die with constant velocity $V_{\square}$ without any further deformation. The volume of the material does not change before and after extrusion, so the speed relationship of the material in region $\square$ and region $\square$ can be expressed as $V_{\square}=$ (b/a) $V_{\square}$. Region $\otimes$ is the first deformation zone, which is separated from region I and region III by entry and exist surfaces $\Gamma_{1}$ and $\Gamma_{2}$. They belong to the slip line field of first deformation zone (DZ-1) and correspond to the beginning and end of deformation. Similarly, $\Gamma_{3}$ and $\Gamma_{4}$ are the entry and exist surfaces of the second deformation zone (DZ-2), which belong to the slip line field of DZ-2.

Slip line field (SLF) analysis is a traditional theoretical method to analyze metal forming characteristics. With the SLF solution, the extrusion mechanism through wedge-shaped die has been well studied by many scholars ${ }^{[2,21]}$. Due to the similarity of die apertures, the SLF of AGE can be deduced which is illustrated in Figure 3. As discussed above, two independent deformation zones (DZs) should be formed in AGE, and correspondingly there are two isolated SLFs distributed along channel vertical direction.

As shown in Figure 3, the vertical distribution of these two SLFs reveals that the parameter $h$ largely defines the behavior between these two DZs. In order to form two independent DZs, a large value should be used for $h$. Due to the SLFs of these two DZs are very similar in characteristic, only the SLF of DZ-1 is discussed in detail below. The geometry of DZ-1 slip line configuration is defined by the field angles $\lambda_{1}$, $\varphi_{1}$, and $\theta_{1}$ and the die angle $a$. The value of $\lambda_{1}$ is dependent on the friction condition at the interface between the die and the work material. As for a frictionless condition, the value of $\lambda_{1}$ equals $\pi / 4$. The parameter $\varphi_{1}$ reflects the range of reductions for which the field is valid. When the extrusion ratio tends to its maximum value (for which $\theta_{1}=0$ ), the SLF tends to its limit. When $\theta_{1} \rightarrow 0$, the point $E_{1} \rightarrow D_{1}$, and the point $C_{1} \rightarrow F_{1}$ so that the slip line field $A_{1} E_{1} C_{1} F_{1} B_{1}$ can be approximated with a triangular one $A_{1} B_{1} C_{1}$. The kinematically admissible velocity field associated with the SLF from DZ-1 becomes a triangular velocity field as shown in figure 4(a). In this field, the material moves parallel to the inclined wall with velocity $V_{\mathrm{a}}$. In each point on the surfaces $\Gamma_{1}$ and $\Gamma_{2}$ the velocities $V_{\square}$ and $V_{a}$ suddenly change the direction.

For an arbitrary point $\mathrm{P}$ on $\mathrm{AC}$, its corresponding point on $\mathrm{BC}$ is $\mathrm{Q}$. As shown in Figure 2, the velocity at point $\mathrm{P}$ is affected by both the region $\otimes$ velocity $V_{\square}$ and region $\otimes$ velocity $V_{\mathrm{a}}$. The velocity decomposition of $V_{\square}$ and $V_{\mathrm{a}}$ along the $\mathrm{AC}$ tangential and normal directions is shown in the figure 4(a). Due to the continuity of metal flow, $V_{\square}$ and $V_{\mathrm{a}}$ on the AC normal direction must be equal, that is $V_{\text {an_AC }}=V_{\text {区n }}$. However, as illustrated in the Figure 4(a), the velocities of $V_{\square}$ and $V_{a}$ on the AC tangential direction have different values, $V_{\text {at_AC }}>V_{\mathbb{Q D t}}$. The differences between $V_{\text {at_AC }}$ and $V_{\mathbb{}}$ define the velocity discontinuities, giving rise to tangential stress $\tau_{\mathrm{AC}}$. Similarly, at point $\mathrm{Q}$ on $\mathrm{BC}$, the velocity decomposition of region $\nabla$ velocity $V_{\mathrm{a}}$ and region $\otimes$ velocity $V_{\square}$ in the $\mathrm{BC}$ normal and tangential directions is also displayed in the Figure 4(a). In addition, the velocity $V_{\text {at_BC }}$ and $V_{\mathbb{~} \mathrm{t}}$ in the $\mathrm{BC}$ tangential direction are also different. Therefore, a stress $\tau_{\mathrm{BC}}$ along the $\mathrm{BC}$ tangential direction is formed at point $\mathrm{Q}$. 
The tangential stress distribution on the boundary of the two deformation zones in AGE is schematic illustrated in Figure 4(b). When an elongated and thin enough grain (obtained from a previous AGE pass) crosses one velocity discontinuity surface along which tangential stress exceeds strength in pure shear $(\tau$ $>\mathrm{k}$ ), the grain strength will be overcome and the fragmentation process starts. Accordingly, the speed discontinuous surface plays an important role in grain fragmentation. The larger the number of discontinuous surfaces, the more effective the fragmentation is. Compared with traditional extrusion, AGE forms two independent DZs, increasing the number of discontinuous surfaces. Moreover, the shear stresses on the discontinuous surfaces of the two DZs are complementary. The uniformity of the shear stress formed by AGE is greatly enhanced.

\section{Finite Element Analysis}

Table 1. Parameters used in finite element models

\begin{tabular}{cccccc}
\hline & $a / m m$ & $b / m m$ & $\alpha / \operatorname{deg}$ & $\beta / \operatorname{deg}$ & $h / m m$ \\
\hline Model-1 & 10 & 15 & 15 & 15 & 0 \\
Model-2 & 10 & 15 & 15 & 15 & $2 *[(b-a) / 2] / \tan \alpha$ \\
Model-3 & 10 & 15 & 15 & 15 & {$[(\mathrm{~b}-\mathrm{a}) / 2] / \tan \alpha$} \\
\hline
\end{tabular}

To verify the deformation characters of AGE as discussed in section 2, finite element analysis is proposed in this section. In order to highlight the influence of parameter $h$, three analysis models with different value for $h$ were established. These three analysis models are named Model-1, Model-2 and Model-3 respectively. Corresponding to the parameters of AGE as illustrated in Figure 1(b), their values used in the three finite element models are listed in Table 1. The first model which named Model-1 is symmetry extrusion as the value 0 is used for $h$. In addition, the other models which named Model-2 and Model-3 are all asymmetry extrusion. However, a higher value $h$ has been used in Model-3 which leads to generating two independent DZs. Distinguish with Model-3, the two DZs are interacted in Model-2 as a small value is assigned for $h$.

The commercial finite element software DEFORM was used for simulation. As discussed in section 1, all the simulations are proceed based on plane strain assumption. The punch and die are all set as rigid, and an elastic-plastic model is used for workpiece. A material AL-1100 was chose for workpiece from the DEFORM material library. Shear friction model with a coefficient 0.12 was used for all the contact surfaces. The punch moves along channel vertical direction with a velocity of $1 \mathrm{~mm} / \mathrm{s}$, and a room temperature $20^{\circ} \mathrm{C}$ was assigned for all simulations. Workpieces used in the three analysis modes with the same geometry dimensions and meshes, which are all meshed by mapping method and plane strain elements.

\subsection{Characters of the deformation zones}


Variation of mesh shape can be regarded as an indirect reflection of deformation characteristics. The results of mesh distortion with the three analysis models in extrusion process are shown in Figure 5. The significant influence of parameter $h$ on the interaction manners of DZs is schematically verified. In Model1 , two DZs are formed symmetrically about the vertical center of workpiece. With increasing $h$, the two DZs are gradually separated on the extrusion direction (Model-3). In addition, two independent DZs would be generated as a larger value has been assigned to $h$ (Model-2). As these two independent DZs forming, a dead metal zone where materials flow rigidly is generating simultaneously. In terms of the total area of DMs in workpiece, Model-2 takes the first place in value. It can be deduced that AGE (Model-2) is more efficient in workpiece deformation than other conventional extrusions under one pass.

The results of velocity field in DZs of workpiece for these three analysis models are displayed in Figure 6. As discussed in section 2, due to constancy of volume, materials at die outlet should be of the same velocity as an identical moving condition implied on punches in the three analysis models. Corresponding to the results shown in Figure 5, Model- 1 which is a conventional symmetric extrusion has the smallest velocity variation area among the three models. Through transforming the symmetrical structure into vertical separation of channel, a larger velocity field can be formed as displayed in Model-2 and Model-3. However, the Model-2 which is an AGE emphasized in this article produces two independent velocity variation areas. It is meaningful that materials can be shaped more smoothly in AGE, and forming defects such as cracks can be largely restrained.

Slip line field in workpiece can be reflected by the simulation results of equivalent strain rate which are displayed in Figure 7. According to the results, a higher strain rate has been generated at the entrance and exit of each deformation zones. With a larger parameter $h$ is assigned, two independent DZs are formed in AGE (Model-2). It means that materials would be suffered shear effects with four times in one pass. In other words, more works can be impacted on grains fragment. Through AGE, the efficiency of extrusion on grains refinement has be largely improved. With simulation results of equivalent strain rate, the correctness of slip line field as analyzed and discussed in section 2 has been verified.

\subsection{Comparison of extrusion results}

The contour plot of equivalent strain distribution in workpiece after extrusion is shown in Figure 8. The results show that for the symmetric extrusion of Model-1, the formed equivalent strain field also presents a symmetric distribution. In addition, the equivalent strain field formed by asymmetric extrusion, Model-2 and Model-3, is larger than that by symmetric extrusion in value. However, Model-2 which forms two independent plastic deformation zones, has more uniform distribution of equivalent strain than Model 3.

The results of equivalent stress distribution along the midline $O P$ of workpieces are shown in Figure 9. Corresponding to the characteristics of deformation, the equivalent strain formed by Model- 1 is symmetrically distributed. The equivalent strain in two asymmetric extrusion, Model-2 and Model-3, is larger than that in symmetric extrusion with Model-1. In addition, the equivalent strain distribution formed by Model 2 is the most uniform, and its equivalent strain fluctuation is less than 0.05 . It should be 
emphasized that for asymmetric extrusion, the extrusion angles of two deformation zones can be independently adjusted to obtain higher and more uniform equivalent strain results.

The statistical results of the equivalent strain in workpieces after extrusion is shown in Figure 10. It can be seen from the results that the equivalent strain distribution of Model- 2 is the most concentrated. The average values of equivalent strain of the three models are $0.518,0.638$ and 0.607 respectively. All the analysis results show that the asymmetric extrusion with two independent plastic deformation zones, Model-2, can form large and uniform equivalent strain in workpiece. Therefore, the advantage of asymmetric extrusion proposed in this paper in the preparation of ultrafine-grained materials has been verified.

\section{Conclusions}

A novel method for preparing ultrafine-grained materials which named asymmetry direct extrusion is presented in this article. In addition, the deformation characters of this process were analyzed by slip line field and finite element method. Compared with the traditional extrusion method, AGE can form two independent deformation zones. Moreover, these two deformation zones are complementary. The distance between the two deformation zones is a key parameter. Too small distance will cause mutual interference between the two deformation zones and deteriorate the uniformity of deformation. The results of strain analysis after extrusion show that, compared with other extrusion methods, the internal strain after asymmetric extrusion is large and uniform. Asymmetric Gradient Extrusion has shown great advantages in the preparation of bulk ultrafine-grained materials.

\section{Declarations}

\section{Ethical Approval}

The article follows the guidelines of the Committee on Publication Ethics (COPE) and involves no studies on human or animal subjects.

\section{Consent to Participate}

Not applicable. The article involves no studies on humans.

\section{Consent to Publish}

Not applicable. The article involves no studies on humans.

\section{Authors Contributions}

Junkai Fan and Chengpeng Wang conceived and designed the simulation process; Junkai Fan and Wu Zhao performed the simulations; Junkai Fan analyzed the data and wrote the paper; Wei Liu reviewed the paper. 


\section{Funding}

This work is partially supported by the National Key R\&D Program of China (grant No. 2019YFC0408400), Science and Technology Research Fund of Henan Provincial Science and Technology Department (grant No.212102210004 and No. 202102210108), Shandong Province Key R\&D Program (grant No.2019TSLH0304), Young Backbone Teachers Training Program of Henan Polytechnic University (grant No.2020XQG-01) and Henan Polytechnic University Innovation Team Project (grant No.T2019-5).

\section{Conflict of Interest}

The authors declare that they have no conflict of interest.

\section{Availability of data and materials}

All the data have been presented in the manuscript.

\section{References}

1. Yang C, Song N, Liao X (2018) Structural evolutions of metallic materials processed by severe plastic deformation. Materials Science Engineering: Reports 133:1-59

2. Sadasivan N, Balasubramanian M (2021) Severe plastic deformation of tubular materials -Process methodology and its influence on mechanical properties-A review. Materials Today: Proceedings 2021(9)

3. Horita Z, Furukawa M, Nemoto M (2000) Development of fine-grained structures using severe plastic deformation. Mater Sci Technol 16(11-12):1239-1245

4. Kawasaki M, Langdon T (2016) Review: achieving superplastic properties in ultrafine-grained materials at high temperatures. Journal of Materials Science 51(1):19-32

5. Figueiredo RB, Langdon TG (2012) Fabricating Ultrafine-Grained Materials through the Application of Severe Plastic Deformation: a Review of Developments in Brazil. Journal of Materials Research Technology 1(1):55-62

6. Valiev RZ, Estrin Y, Horita Z (2016) Producing Bulk Ultrafine-Grained Materials by Severe Plastic Deformation: Ten Years Later. JOM 68(4):1216-1226

7. Edalati K, Horita Z (2016) A review on high-pressure torsion (HPT) from 1935 to 1988. Materials Science Engineering A 652:325-352

8. Saito Y, Tsuji N, Utsunomiya H (1998) Ultrafine grained bulk aluminum produced by accumulative roll-bonding (ARB) process. Scripta Mater 39(9):1221-1227

9. Kamikawa N, Tsuji N, Huang X, Hansen N (2006) Quantification of annealed microstructures in ARB processed aluminum. Acta Mater 54(11):3055-3066

10. Bahmani A, Arthanari S, Shin KS (2019) Improved corrosion resistant and strength of a magnesium alloy using multi-directional forging (MDF). The International Journal of Advanced Manufacturing 
Technology 105(1-4):785-797

11. Nakao Y, Miura H (2011) Nano-grain evolution in austenitic stainless steel during multi-directional forging. Materials Science Engineering A 528(3):1310-1317

12. Zughaer HJ, Nutting J (2013) Deformation of sintered copper and 50Cu-50Fe mixture to large strains by cyclic extrusion and compression. Metal Science Journal 8(12):1104-1107

13. Babaei A, Mashhadi MM (2014) Characterization of ultrafine-grained aluminum tubes processed by Tube Cyclic Extrusion-Compression (TCEC). Mater Charact 95:118-128

14. Gtuchowski W, Stobrawa J, Rdzawski Z (2011) Ultrafine Grained Copper Alloys Processed by Continuous Repetitive Corrugation and Straightening Method. Mater Sci Forum 674:177-188

15. Me A, laf A, Se B (2020) Numerical and experimental study of a 5754-aluminum alloy processed by heterogeneous repetitive corrugation and straightening. Journal of Materials Research Technology 9(2):1941-1947

16. Kalahroudi FJ, Eviani AR, Jafarian HR, Amouri A, Gholizadeh R (2016) Inhomogeneity in strain, microstructure and mechanical properties of AA1050 alloy during twist extrusion. Materials Science Engineering A 667:349-357

17. Asghar SA, Mousavi A, Bahador SR (2011) Investigation and numerical analysis of strain distribution in the twist extrusion of pure aluminum. JOM 63(2):69-76

18. Lee JW, Park JJ (2002) Numerical and experimental investigations of constrained groove pressing and rolling for grain refinement. J Mater Process Technol 130:208-213

19. Yoon SC, Krishnaiah A, Chakkingal U (2008) Severe plastic deformation and strain localization in groove pressing. Comput Mater Sci 43(4):641-645

20. Zaharia L, Chelariu R, Comaneci R (2012) Multiple direct extrusion: A new technique in grain refinement. Materials Science Engineering A 550:293-299

21. Zaharia L, Comaneci R, R.Chelariu (2014) A new severe plastic deformation method by repetitive extrusion and upsetting. Materials Science Engineering: A 595(1):135-142

22. Lin HK, Huang JC (2002) High strain rate and/or low temperature super plasticity in AZ31 Mg alloys processed by simple high-ratio extrusion methods. Mater Trans 43(10):2424-2432

23. Hwang YM, Yang MT (2002) Study of Hydrostatic Extrusion Processes with Extra-High Extrusion Ratio. Key Eng Mater 233:311-316

\section{Figures}




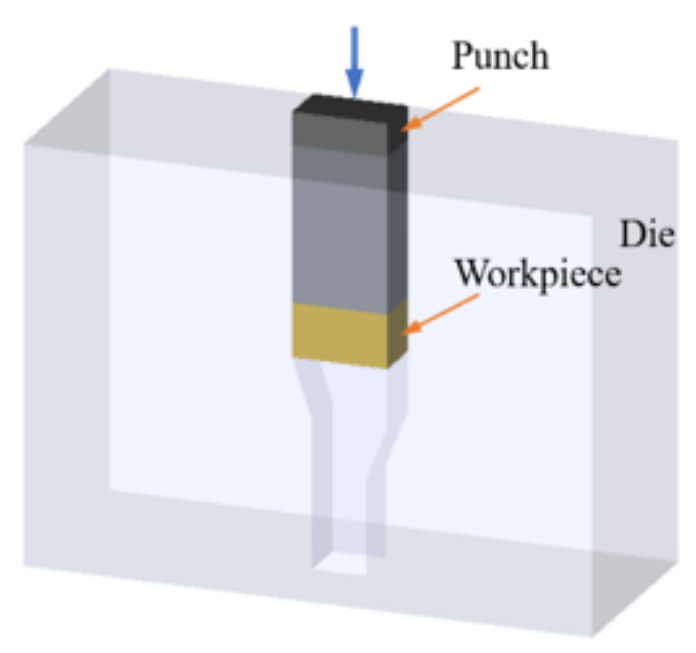

(a)die and workpiece

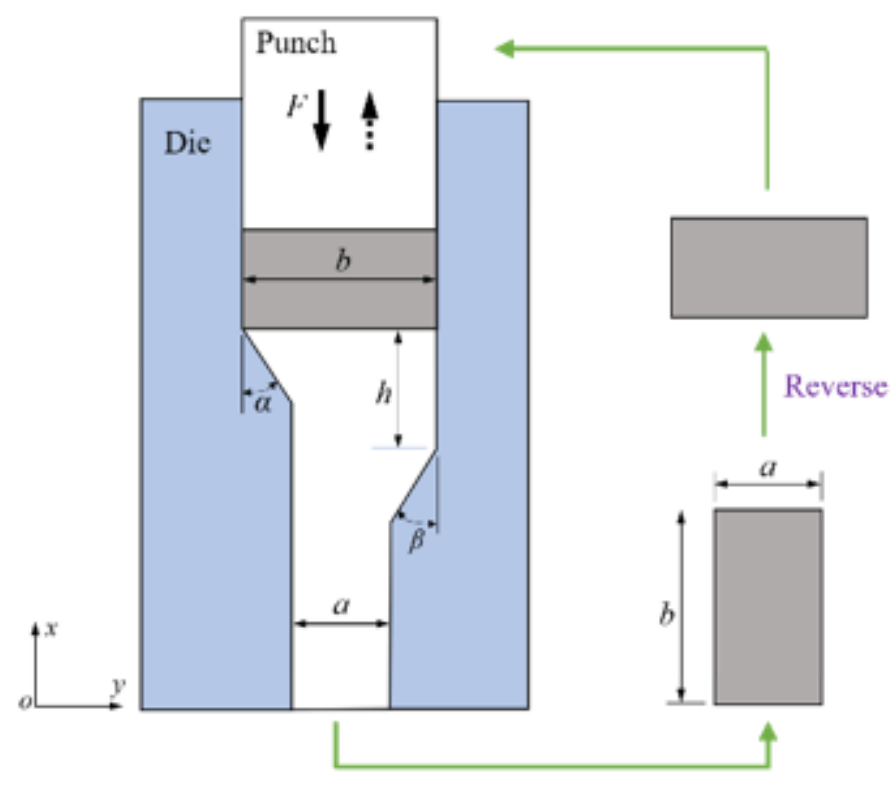

(b)schematic representation of AGE process

Figure 1

Principle of AGE technique

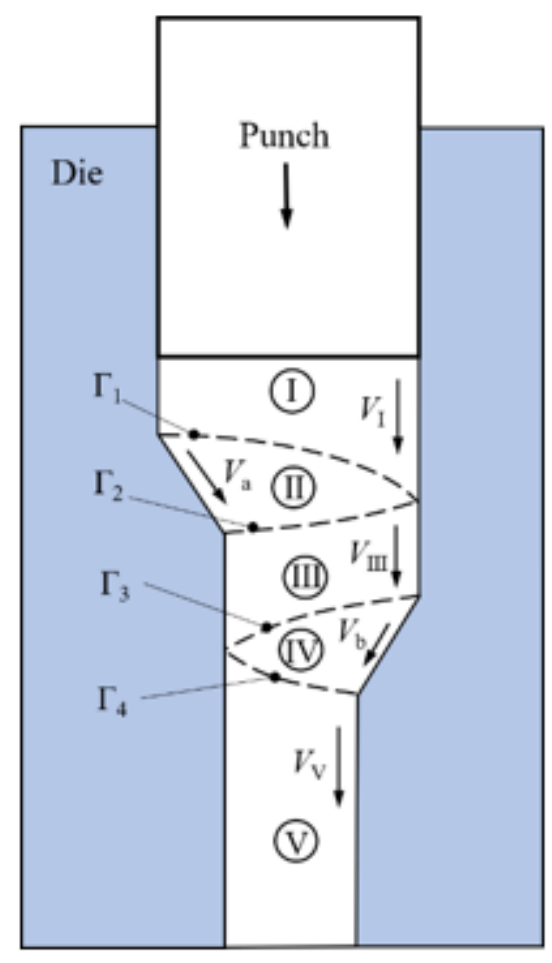

Figure 2

Typical deformation regions formed in AGE 


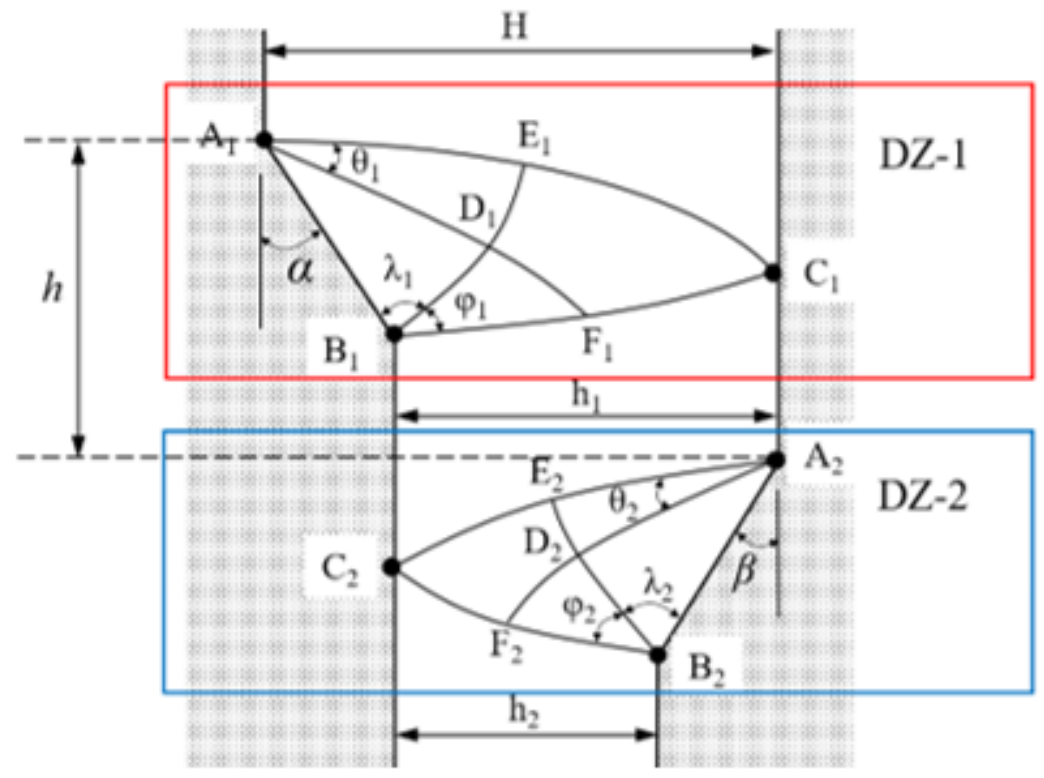

Figure 3

Slip line fields of AGE

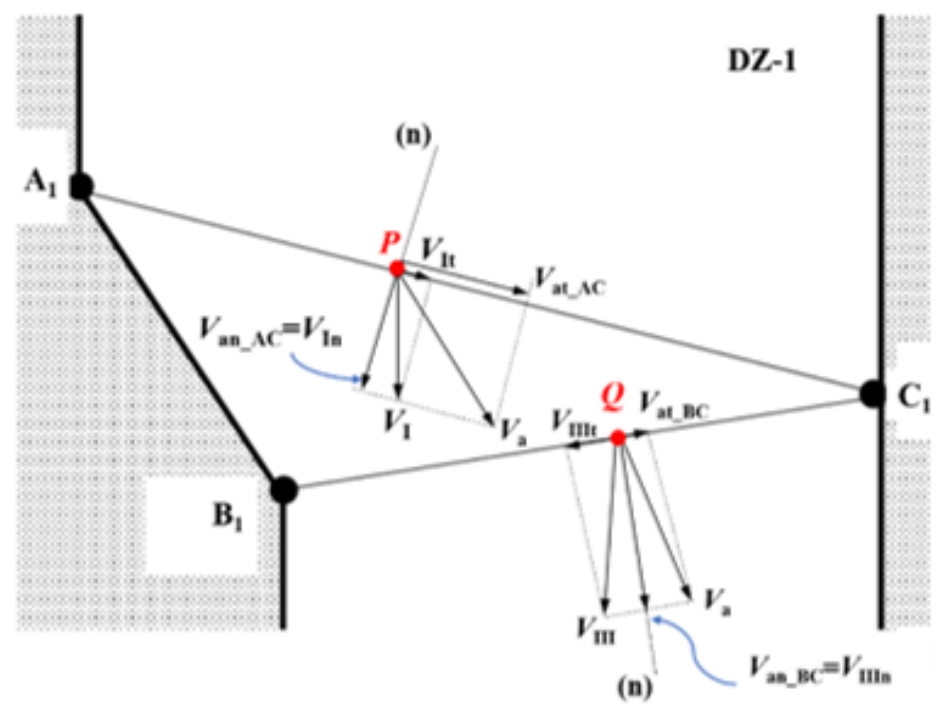

(a)components of velocities on slip lines

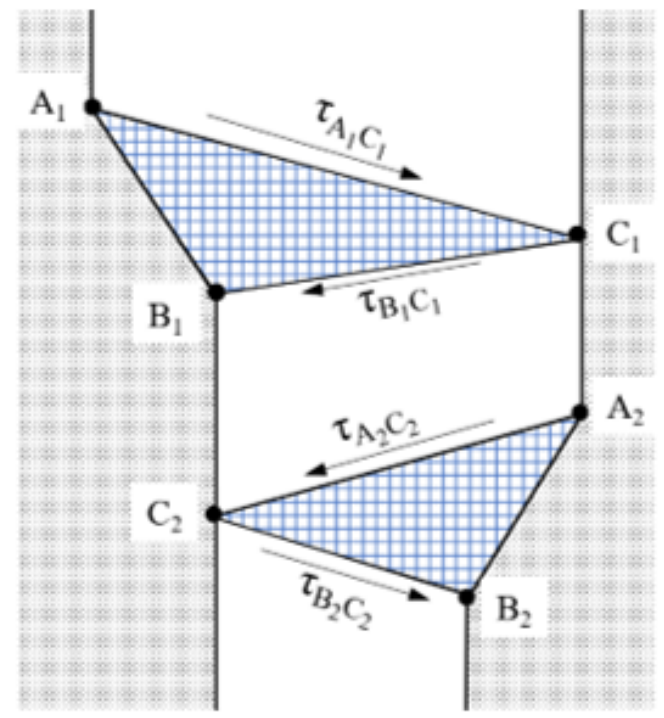

(b)two shear zones in AGE

Figure 4

Shear characteristics of deformation zones in AGE 

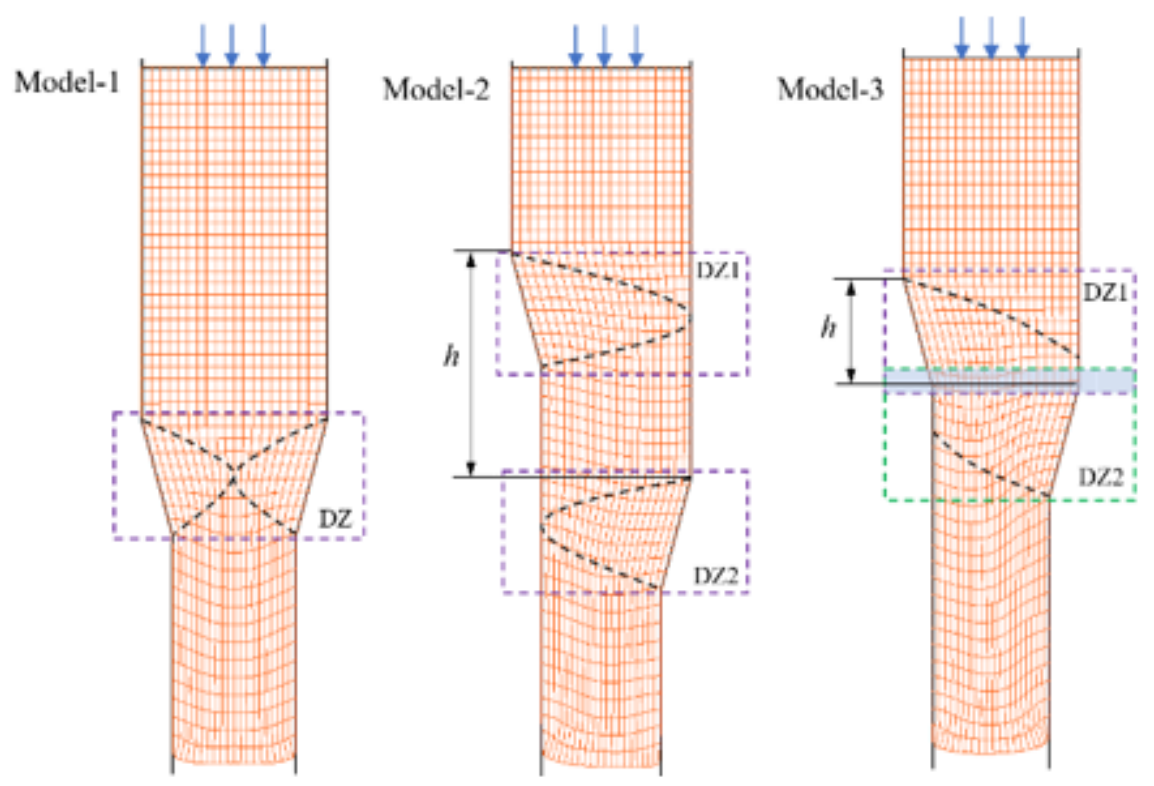

Figure 5

Distortion of meshes in deformation zones

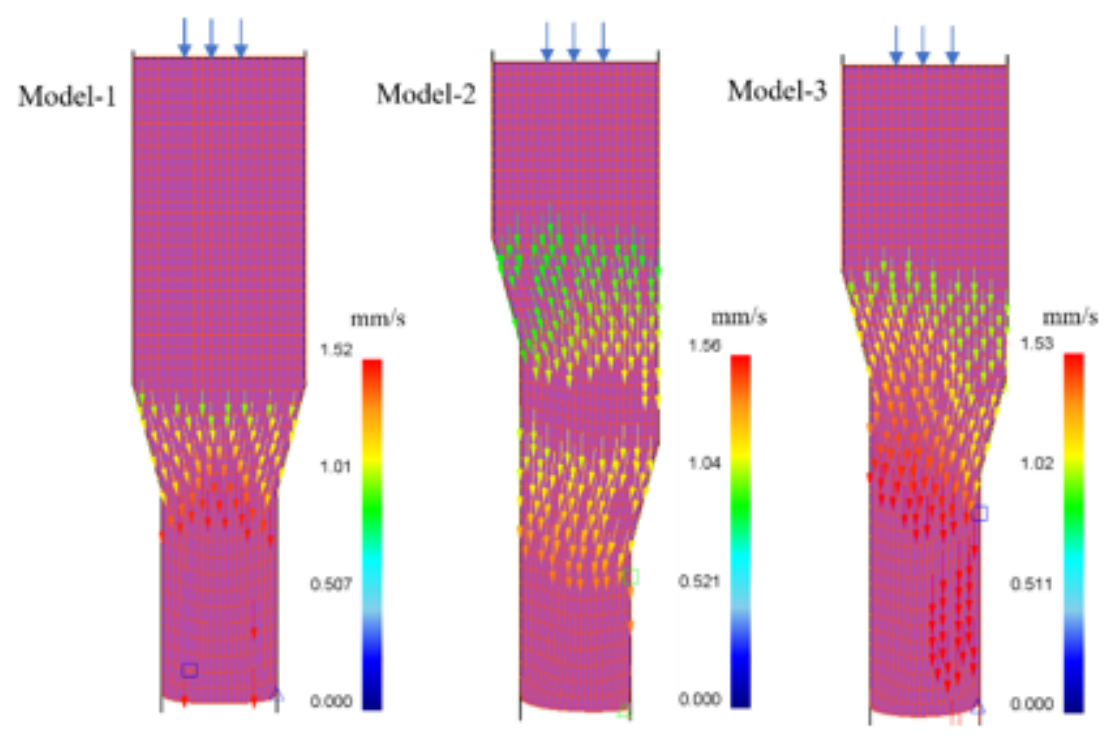

Figure 6

Velocity field of deformation zones 


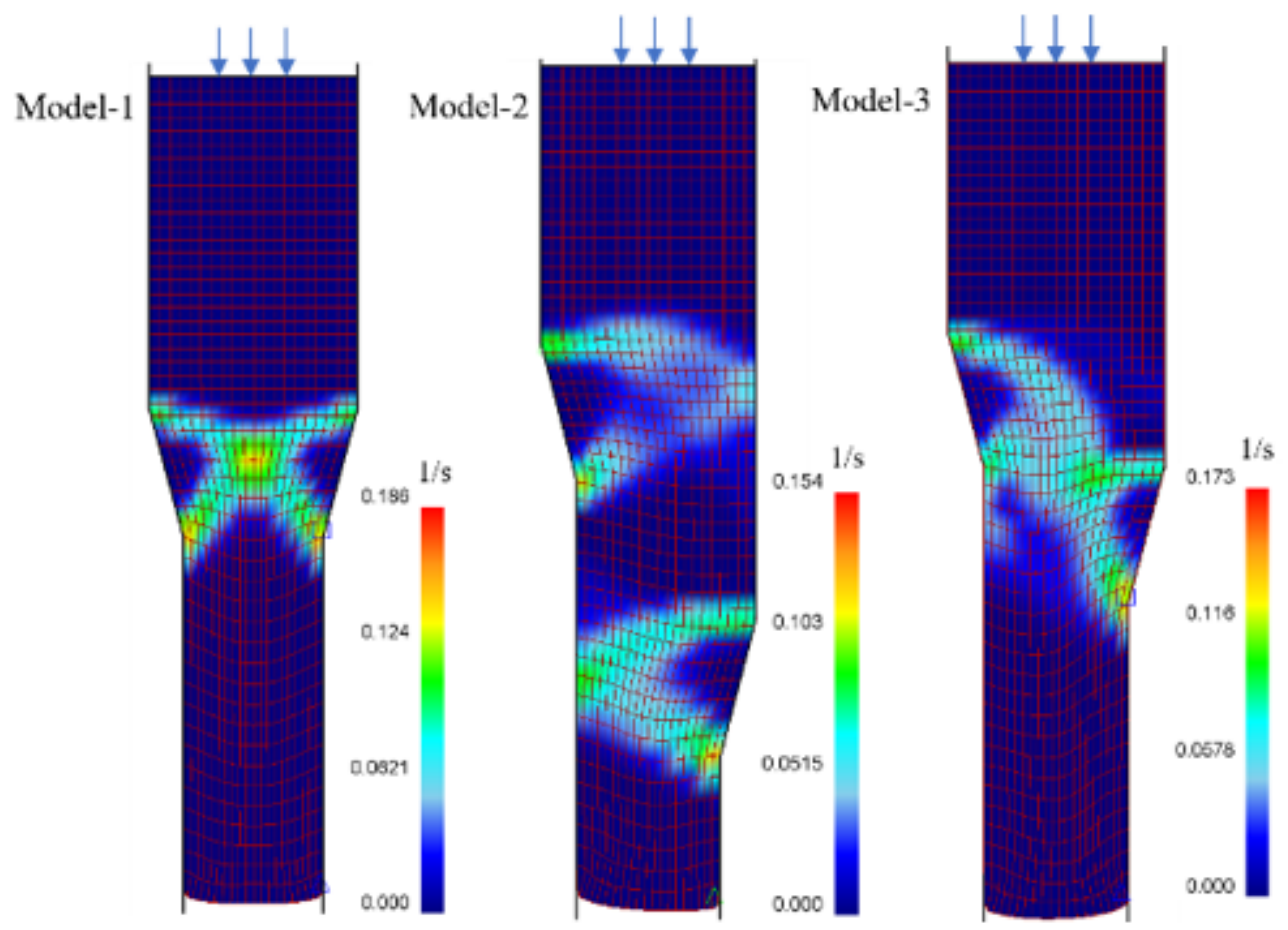

Figure 7

Equivalent strain rate during extrusion

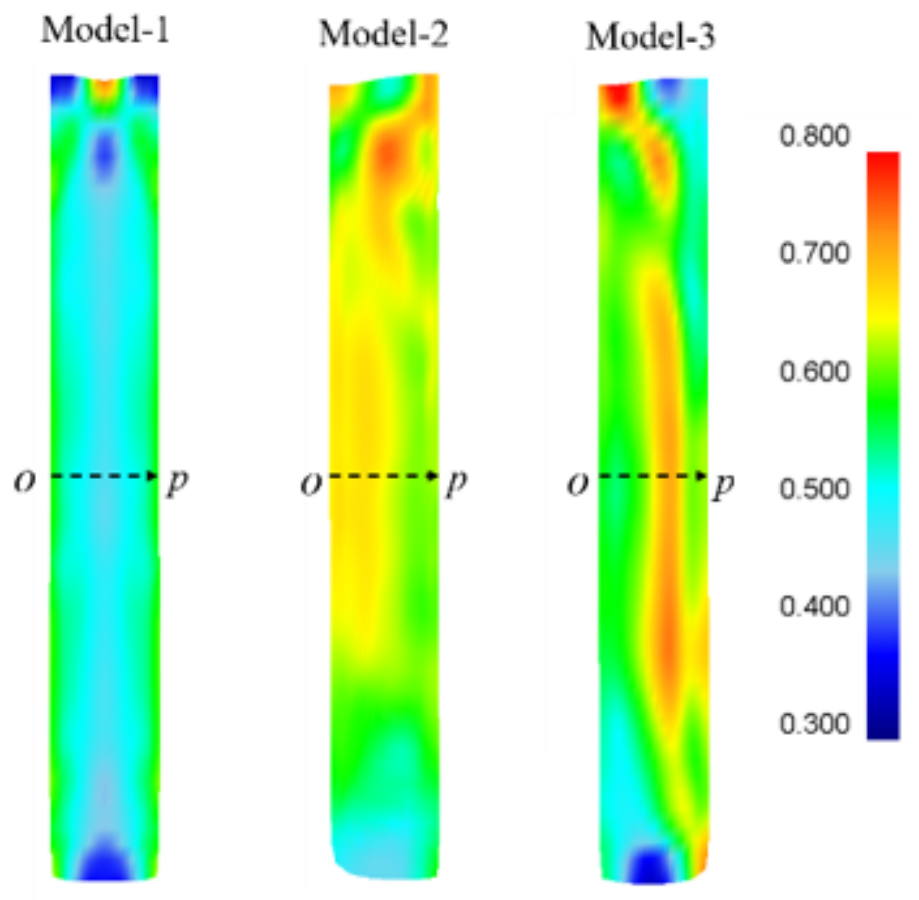

Figure 8

Contour plot of equivalent strain in the workpiece after extrusion 


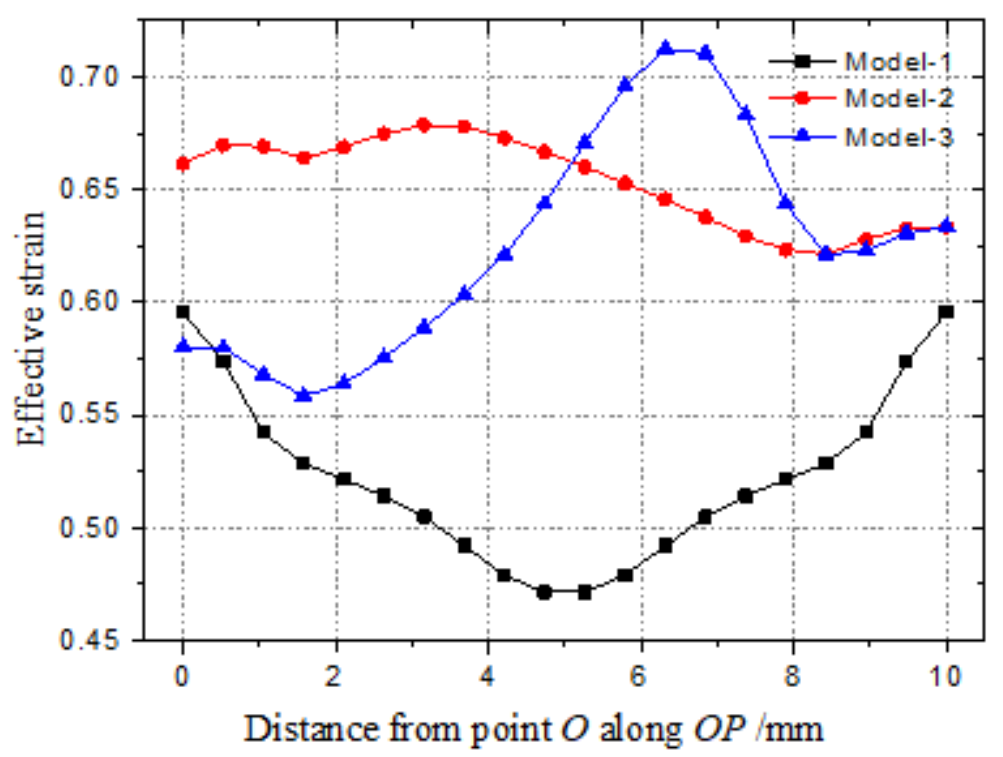

Figure 9

Equivalent strain distribution along OP line

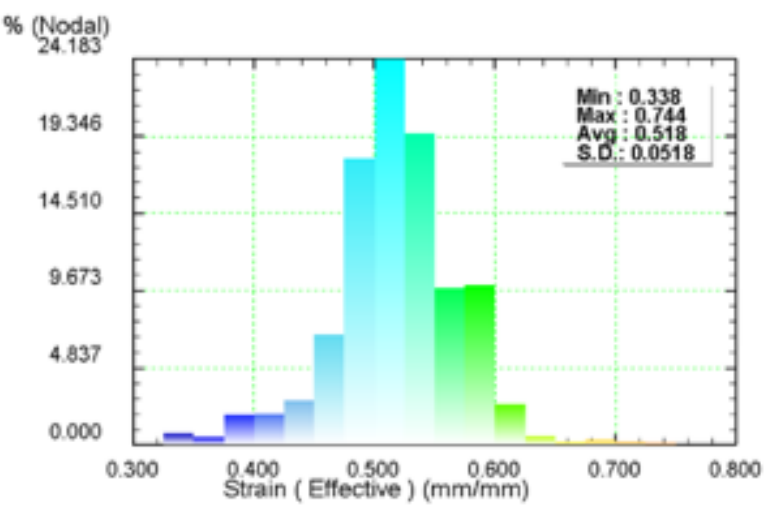

(a) Model-1

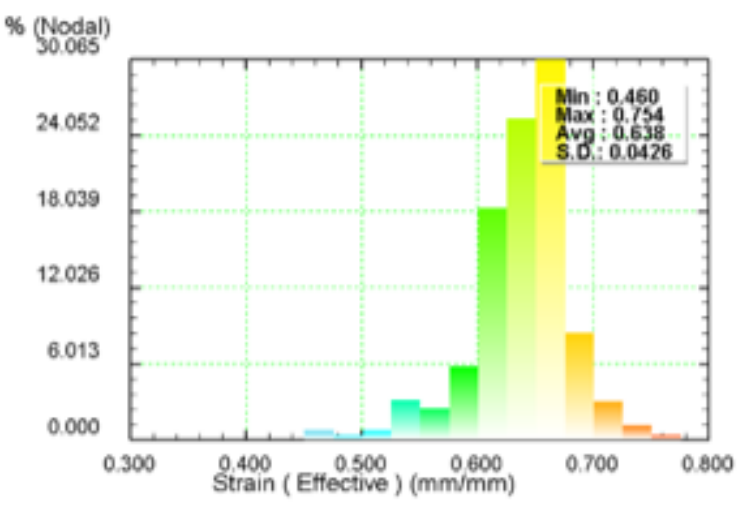

(b) Model-2

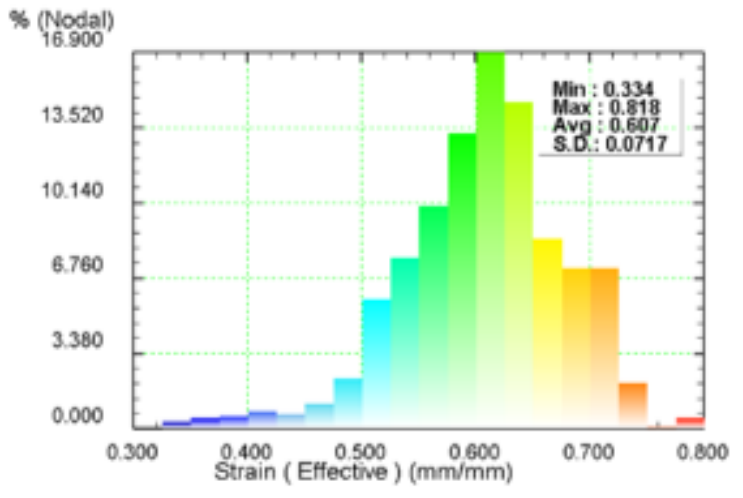

(c) Model-3 
Figure 10

Statistical results of the equivalent strain in workpieces after the extrusion 